\title{
Genomic Instability: The Cause and Effect of BCR/ABL Tyrosine Kinase
}

\author{
Tomasz Skorski, MD, PhD, DSc
}

\author{
Corresponding author \\ Tomasz Skorski, MD, PhD, DSc \\ Department of Microbiology and Immunology, School of Medicine, \\ Temple University, MRB 548A, 3400 N. Broad Street, Philadelphia, \\ PA 19140, USA. \\ E-mail: tskorski@temple.edu
}

Current Hematologic Malignancy Reports 2007, 2:69-74

Current Medicine Group LLC ISSN 1558-8211

Copyright (C) 2007 by Current Medicine Group LLC

Genes encoding $\mathrm{c}-\mathrm{ABL}$ kinase and BCR protein are targeted by yet-unknown mechanisms causing DNA double-strand breaks resulting in the generation of a chimeric gene encoding $\mathrm{BCR} / \mathrm{ABL}$ fusion tyrosine kinase. $\mathrm{BCR} / \mathrm{ABL}$ kinase displays transforming activity because of its constitutive kinase activity causing deregulated proliferation, apoptosis, differentiation, and adhesion. Moreover, BCR/ABL kinase is able to facilitate DNA repair, prolong activation of G2/M and $S$ cell cycle checkpoints, and elevate expression of the antiapoptotic protein Bcl- $X_{L^{\prime}}$ making malignant cells less responsive to antitumor treatment. BCR/ABL may also stimulate generation of reactive oxygen species and enhance spontaneous DNA damage in tumor cells. Unfortunately, BCR/ABL kinase compromises the fidelity of DNA repair mechanisms, thus contributing to the accumulation of additional genetic abnormalities that lead to resistance to inhibitors such as imatinib mesylate and to malignant progression of the disease. Therefore, chronic myelogenous leukemia cells display mutator phenotype.

\section{Introduction}

There are 90 tyrosine kinase (TK) genes known to date [1], encoding a cohort of catalytically active molecules that are tightly regulated by interaction with their ligands and inhibitors and by phosphorylation/dephosphorylation [2]. TK genes are targeted chronically by genetic errors, which lead to the generation of mutated enzymes exerting uncontrolled activity. At least three mechanisms may lead to constitutive activation of the catalytic activity: chromosomal translocation, overexpression, and activating mutations [3]. These changes cause perturbed signal transduction eventually resulting in neoplastic disease.

$B C R / A B L$ is a result of a $\mathrm{t}(9 ; 22)$ reciprocal translocation of a segment of the $c-A B L$ gene from chromosome 9 to a portion of the $B C R$ gene locus on chromosome 22 . It is present in chronic myelogenous leukemia (CML) and in a cohort of patients with acute lymphoblastic leukemia (ALL) [4].

\section{$B C R / A B L$ Formation}

Genetic anomalies, resulting from unfaithful repair of elevated levels of DNA double-strand breaks (DSBs) may appear even before cancer is clinically diagnosed $[5,6 \bullet]$. Chromosomal translocations arise as a result of the fusion (misrepair) of two or more broken ends of chromosomes that occur in spatial proximity to one another [7]. Elegant studies by Richardson and Jasin [8] demonstrated that two DNA DSBs, each on different chromosomes, are sufficient to promote frequent reciprocal chromosomal translocations. Reactive oxygen species (ROS), ionizing radiation, genotoxic chemicals, and DNA replication stress are likely factors to consider as a source of DSBs. However, what induces the chromosome strands to finally break and why DNA repair mechanisms act improperly to produce chromosomal translocations is open to conjecture.

The cells of the human body must unremittingly counteract constant assault by ROS such as superoxide anions $\left(\mathrm{O}_{2}^{-}\right)$, hydroxyl radicals $(\cdot \mathrm{OH})$, singlet oxygen $\left({ }^{1} \mathrm{O}_{2}\right)$, and hydrogen peroxide $\left(\mathrm{H}_{2} \mathrm{O}_{2}\right)$. It is estimated that about 104 oxidative DNA lesions occur per human cell per day [9]. ROS can be elevated by various physiologic stimuli such as hematopoietic growth factors. In addition, elevated release of ROS by leukocytes is observed during infections and inflammations, which are implicated in the induction of a quarter of all cancer cases worldwide. Moreover, the activation of cellular stress signals from unfavorable conditions such as aberrant oxygen tension may lead to the release of ROS from the mitochondria.

Unrepaired oxidative damage may result in the formation of DSBs [10]. The role of ROS in the generation of DSBs is supported by several observations: 1) cellular oxygen tension causes chromosome breaks [10]; 2) overexpression of c-Myc elevates ROS-mediated DSBs [11]; 3) treatment with $\mathrm{H}_{2} \mathrm{O}_{2}$ induces DSBs [12]; 
4) ROS-dependent DNA single-strand interruptions in replicating chromosomes generate DSBs [13]; and 5) chromosomally unstable cells display persistent oxidative stress [14]. Moreover, translocation breakpoints are GC-rich [15] and oxidative damage is predominantly detected in GC-rich sequences [16]. Thus, it is tempting to speculate that clustered oxidative damage creates multiple DNA lesions in a short DNA fragment, which pose a serious obstacle for DNA repair machinery, resulting in a DSB.

DNA replication stress also can induce DSBs independently of ROS [5]. Common chromatin structures at the breakpoint regions may lead to DSBs and chromosomal translocations [17]. Chromatin structural elements, including topoisomerase II (topo II), DNase I cleavage sites, and scaffold-associated regions (SARs), closely associate with BCR and ABL breakpoints. Topo II and scaffold protein II are essential for chromosome condensation. For example, topo II is a key protein with enzymatic and structural functions, responding to torsional stress of DNA. Therefore, the aberrant activity of these elements may result in DSBs.

Moreover, there is strong evidence of radiation-induced risks for CML. Experimental data, as well as cancer incidence records of atomic bomb survivors, indicated that $B C R / A B L$ translocation might occur after irradiation [18].

Specific recombination mechanisms responsible for generation of the $t(9 ; 22)$ chromosomal translocation are currently unknown. Localization of DSBs may be important for the formation of chromosomal translocations. It has been estimated that approximately $6 \%$ to $13 \%$ of genomic DNA consists of repetitive Alu sequences. DNA sequences of surrounding breakpoint regions involved in the formation of $B C R / A B L$ often revealed homology to $A l u$ [19]. However, Alu sequences are not always close to the sites of recombination, so their direct role in chromosomal translocations is not clear. Alu elements may work as structural modifiers of chromatin organization, because translin-binding sites were identified within the Alu consensus [19]. Translin protein binds chromosomal breakpoint sequences and may promote illegitimate conjunctions to favor chromosomal translocations.

Mammalian cells have multiple pathways to repair DSBs that represent a "clear and present danger" to survival and genomic integrity: homologous recombination repair (HRR), nonhomologous end-joining (NHEJ), and singlestrand annealing (SSA) [20]. Experimental data implicate NHEJ, involving small deletions and microhomology, in the formation of chromosomal translocations [21••]. In accordance, microhomology at the $B C R / A B L$ junction region has been detected [22]. HRR does not appear to be involved in the overwhelming majority of cancerassociated translocations, as breakpoint junctions lack extensive homology, and translocations involving this pathway are not recovered in model systems [23••]. However, another homology-driven pathway, SSA, efficiently generates translocations in experimental models [23••].
Consequences of BCR/ABL Kinase Expression BCR/ABL kinase exhibits two complementary roles in cancer. The first role is stimulation of signaling pathways that contribute to malignant transformation, including proliferation in the absence of growth factors, protection from apoptosis in the absence of external survival factors, and invasion [24]. The second role of BCR/ABL kinase in hematologic malignancies is the modulation of responses to DNA damage, rendering cells resistant to genotoxic therapies and causing genetic instability [25].

\section{Drug resistance}

BCR/ABL-transformed cells demonstrate an enhanced ability to survive genotoxic stress, probably because of enhanced DNA repair, inhibition of proapoptotic pathways, and prolonged S and G2/M checkpoints for extended repair $[25,26]$. These three factors may work in concert to provide the necessary protection from DNA damage-induced apoptosis in tumor cells transformed not only by BCR/ABL but also by other oncogenic kinases [27]. Leukemia cells surviving genotoxic attack may harbor DNA aberrations due to the unfaithful DNA repair, resulting in genomic instability $[28,29 \bullet \bullet, 30]$.

Drug resistance depends on the high expression levels of BCR/ABL suggesting amplification or hyperactivation of signaling pathways, DNA repair mechanisms relevant for response to genotoxic stress, or both [31]. Because drug resistance depends on BCR/ABL kinase activity, it is most likely not due to clonal evolution arising from accumulation of additional genomic aberrations. The expression/activity of $\mathrm{BCR} / \mathrm{ABL}$ kinase may increase in the accelerated phase and blast crisis; therefore, it seems reasonable to speculate that at the later stages of the disease, high levels of BCR/ABL kinase trigger more pronounced resistance. Thus, if selection is involved in the development of resistance to apoptosis induced in CML cells by DNA damage, then it should be associated with the emergence of clones displaying elevated BCR/ABL kinase expression or activity. However, as CML progresses, cells can also accumulate additional genetic abnormalities, which further increase the resistance of these cells to genotoxic treatment; this effect may no longer depend on BCR/ABL catalytic activity.

\section{DNA repair}

DNA lesions emerge in various forms and require different repair mechanisms (Table 1). Damaged bases, mismatches, and adducts are repaired by base excision repair (BER), nucleotide excision repair (NER), and mismatch repair (MMR). BCR/ABL-positive myeloid leukemia cells exhibited elevated NER activity in response to UV radiation [32].

DSBs can be induced by genotoxic drugs, replication forks encountering lesions, or irradiation and are lethal if unrepaired. Three distinct mechanisms repair DSBs: HRR, NHEJ, and SSA [20]. HRR and NHEJ can play a pivotal role in genotoxic drug resistance. HRR involves 


\begin{tabular}{lcc}
\hline \multicolumn{2}{l}{ Table 1. Genotoxic agents, the DNA lesions they induce, and the DNA repair mechanisms required } \\
\hline Inducing agent & DNA lesions & DNA repair \\
Replication errors & Mismatch, insertion, deletion & MMR \\
X-rays, oxygen radicals, alkylating agents & Abasic site, 8-OxoG, single-strand break & BER \\
Ultraviolet light & Photoproduct, pirimidine dimer & NER \\
$\begin{array}{l}\text { X-rays, cytostatic agents, replication forks } \\
\text { encountering lesions }\end{array}$ & Interstrand cross-link, double-strand break & HRR, NHEJ, SSA \\
BER-base excision repair; HRR-homologous recombination repair; MMR-mismatch repair; NER-nucleotide excision repair; \\
NHEJ-nonhomologous end-joining; SSA-single-strand annealing.
\end{tabular}

RAD51-driven invasion and pairing of a single-stranded DNA to the homologous template. Elevated levels of RAD51 have been observed in various tumors and were positively associated with genotoxic-therapy resistance [33]. Leukemias and lymphomas expressing BCR/ABLrelated fusion tyrosine kinases (FTKs), such as TEL/ABL, TEL/PDGFßR, TEL/JAK2, and NPM/ALK display elevated levels of RAD51 and enhanced HRR activity [30,34,35]. Although Deutsch et al. [36] indicated that DNA-PKcs, an important kinase in NHEJ, may be downregulated in CML cells, subsequent reports employing various in vitro and in vivo methods detected enhanced activity of NHEJ in BCR/ABL-transformed leukemia cells and CML patient cells $[29 \bullet \bullet, 30,37,38]$.

\section{Inhibition of apoptosis}

In addition to modulation of DNA repair mechanisms, BCR/ABL also activates another line of defense against genotoxicity. BCR/ABL kinase may stimulate antiapoptotic proteins such as BCL2 and BCL- $\mathrm{X}_{\mathrm{L}}$ and inhibit pro-apoptotic proteins $\mathrm{BAD}$ and $\mathrm{BAX}$, thus preventing the release of cytochrome $c$ from the mitochondria and activation of caspase-3 [27].

\section{Checkpoint activation}

The time allotted for repair is as crucial as the repair mechanisms themselves. Cell cycle checkpoints are responsible for the mediation of phase transitions, activation of repair mechanisms, and the movement of DNA-repair proteins to lesion sites. Studies from our laboratory and others have demonstrated that cells transformed by BCR/ABL exhibit extended activation of the $\mathrm{S}$ and G2/M cell cycle phase checkpoints when exposed to chemotherapeutic drugs and $\gamma$-radiation [26,34,39]. We have recently reported that $\mathrm{BCR} / \mathrm{ABL}$ leukemia cells might display enhanced stimulation of the ATR-Chk1 axis, which plays an important role in the activation of intra-S-phase checkpoint and drug resistance [26]. This observation is in accordance with another report indicating elevated activation of Chk1 kinase in BCR/ABL cells after $\gamma$-irradiation [40]. However, using tetracycline-induced BCR/ABL expression, Dierov et al. [41] reported inhibition of ATR kinase, abrogation of Chk1 phosphorylation, and disruption of the intra-S-phase checkpoint resulting from translocation of $\mathrm{BCR} / \mathrm{ABL}$ kinase to the nucleus in response to genotoxic treatment. We did not observe nuclear relocation of BCR/ABL kinase in response to DNA damage caused by various drugs and irradiation [26]. The reason for this discrepancy is not known but the effect described by Dierov et al. [41] may depend on the BCR/ABL-inducible expression system.

\section{Facilitation of genomic instability}

It is well known that cancers exhibit chromosomal abnormalities such as translocations, deletions, insertions, and amplifications. Such chromosomal anomalies have been observed at various stages of tumor progression, particularly in the later phases such as CML blast crisis [42]. Genomic instability in BCR/ABL-induced leukemias is manifested by the accumulation of chromosomal aberrations and mutations leading to malignant progression of the disease and acquired resistance to small-molecule inhibitors such as imatinib mesylate $\left(\right.$ Gleevec $^{\circledR}$, Novartis Pharmaceuticals, East Hanover, NJ) $[28,43 \bullet]$.

\section{Genetic aberrations leading to malignant progression of the disease}

CML cells accumulate genetic abnormalities during the course of the disease. The aberrations associated with the progression of BCR/ABL-positive CML chronic phase to the aggressive blast crisis include additional chromosomes $(+\mathrm{Ph},+8,+19)$; isochromosome $17 \mathrm{q}$ (associated with the loss of $\mathrm{p} 53)$; reciprocal translocations $(3 ; 21$, associated with the expression of AML-1/EVIVI1, and 7;11, associated with the expression of NUP98/HOXA9 fusion proteins); other translocations and inversions associated with acute myelogenous leukemia and myelodysplasia, including $\operatorname{inv}(3)$ and $\mathrm{t}(15 ; 17)$; loss of heterozygosity at $14 \mathrm{q} 32$; homozygous mutations/deletions of pRBB and p16/ARF; and mutations in p53 and RAS [43•].

Mutations usually result from enhanced DNA damage, deregulated mechanisms of DNA repair, or both. Reports from our laboratory and others have shown that BCR/ ABL leukemia cells display higher levels of spontaneous DNA damage, such as oxidized bases and DSBs caused by

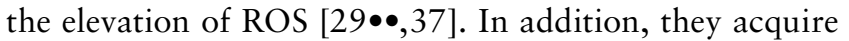
more DNA lesions after genotoxic treatment [26]. As indicated earlier, BCR/ABL may modulate DNA repair 


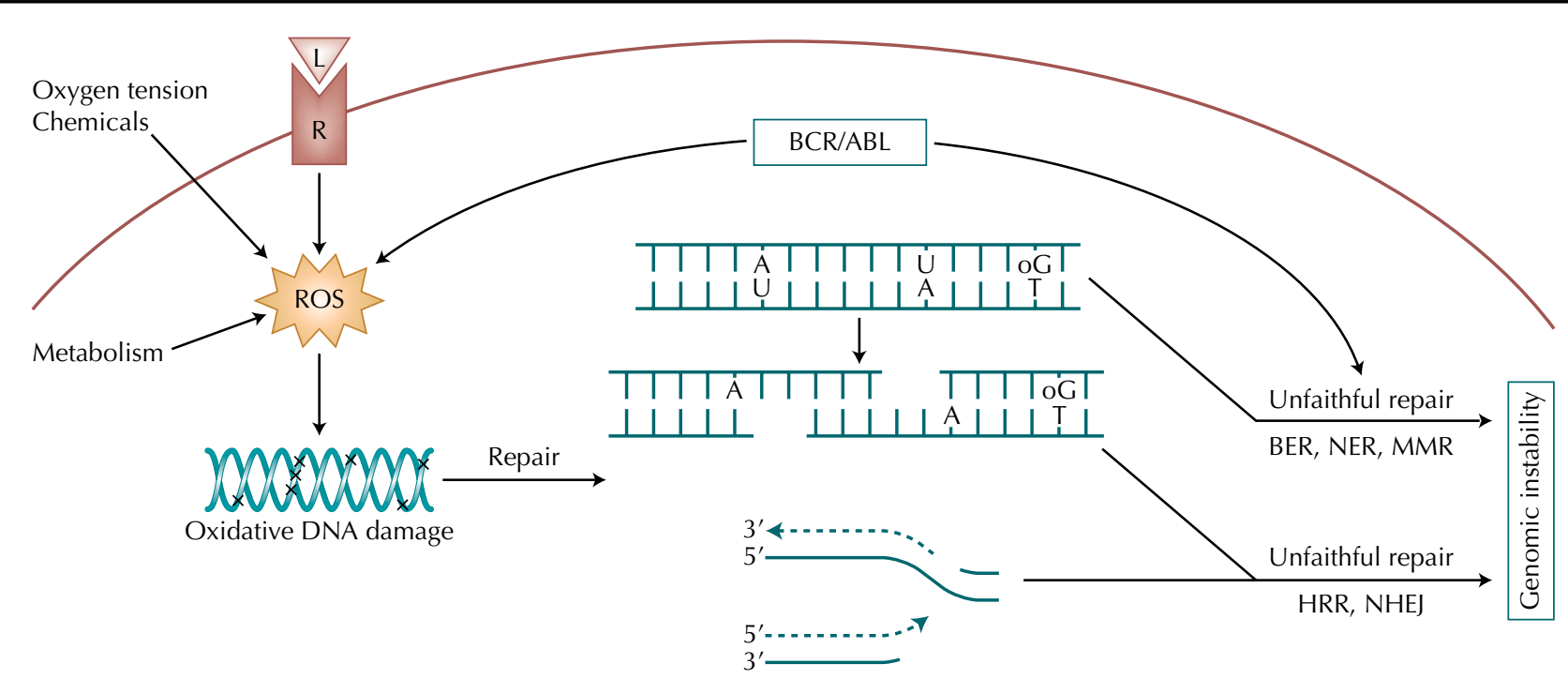

Figure 1. Mechanisms of genomic instability dependent on reactive oxygen species (ROS) in BCR/ABL-positive leukemia cells. Signals from ligand (L)-activated growth factor receptor (R) and other extracellular and intracellular sources stimulate the production of ROS, which cause oxidative DNA damage. Base excision repair (BER), nucleotide excision repair (NER), and mismatch repair (MMR) are involved in the repair of oxidative DNA lesions and prevention of the formation of double-strand breaks (DSBs). DSBs can result from incomplete repair of two oxidative lesions located on different strands at a distance of seven or fewer base pairs, or may occur if the replication fork encounters a nicked oxidative lesion. In leukemia cells, BCR/ABL enhances the production of ROS, resulting in elevated numbers of oxidative DNA lesions, including DSBs. Overexpression of DNA pol $\beta$ can cause base misincorporation during BER and/or homologous recombination repair (HRR), resulting in point mutations. In addition, large deletions are generated during nonhomologous end-joining (NHEJ).

by HRR, NHEJ, and NER, which, in combination with cell cycle arrest and protection from cytochrome $c$-dependent apoptosis, eventually promotes survival [25]. Unfortunately, the repair mechanisms are unfaithful. For example, BCR/ ABL stimulates HRR and NHEJ, but large deletions and numerous point mutations, respectively, were found in the repair products $[29 \bullet \bullet, 30,38]$. Reasons for repair infidelity in BCR/ABL-positive cells are currently unknown.

RAD51 plays a fundamental function in HRR, which is generally an accurate process that repairs DSBs without intermediary deletions, insertions, or chromosomal arrangements [44]. However, increased RAD51 expression not only enhances HRR efficiency to cause drug resistance, but also promotes crossing over, involving gene conversion associated with an exchange of flanking markers leading to chromosomal translocations [45••]. Increased RAD51 also promotes aneuploidy and multiple chromosomal rearrangements. In addition, error-prone DNA polymerases, such as polymerase $\beta$, which exhibits elevated expression in BCR/ABL cells [46], may eventually replace other polymerases and introduce mismatches during HRR. Interestingly, base misincorporations made during DSB repair in Saccharomyces cerevisiae were not substrates for the MMR machinery [47]. Therefore, mismatched bases incorporated into HRR sites might not be removed efficiently, causing mutations in the recombination products. In conclusion, these data provide a link between elevated RAD51 protein levels, genomic instability, and tumor progression.

The molecular explanation for extensive degradation of DSBs preceding NHEJ in BCR/ABL cells is unknown.
Aberrantly regulated exonucleases like ExoI, Mre11, and Artemis could be suggested as potential mediators of this effect [48]. Moreover, NER in p210 BCR/ABL-positive cells was associated with mutations in the HPRT gene. Altogether, these observations implicate an excess of DNA lesions and faulty DNA repair mechanisms in malignant progression of CML (Fig. 1).

\section{Mutations in BCR/ABL leading to resistance to kinase inhibitors}

Imatinib mesylate (IM), a selective inhibitor of the ABL kinase activity, revolutionized the treatment of CML [49]. However, clinical and experimental observations reveal that resistance to the drug has become a rising problem [28]. Resistance may be achieved by enhanced expression of the kinase, blocking of the drug, reduction of cellular concentration of the drug, or mutations in the $B C R / A B L$ gene. $B C R / A B L$ gene mutations appear to play a major role in IM resistance of leukemias positive for the Philadelphia chromosome. Novel inhibitors are being generated to overcome IM resistance, but mutations causing resistance against new drugs are likely to emerge. The mechanisms causing mutations are poorly characterized.

Our recent studies implicate ROS-induced oxidative DNA damage as a source of mutations in the $B C R / A B L$

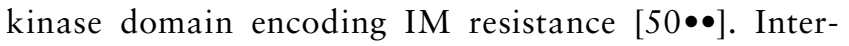
estingly, a GC-rich region is present in the $B C R / A B L$ kinase and is a "hot spot" for ROS-induced DSBs. Moreover, we showed that point mutations might be introduced during HRR and NER in BCR/ABL- 
positive cells $[29 \bullet \bullet, 30,32]$. Therefore, clustered oxidative damage in a GC-rich region may cause a DSB in the sequence encoding BCR/ABL kinase, whereas DNA polymerase-mediated mispairing opposite to an oxidized base during HRR-dependent DNA replication may be responsible for the mutagenic effect. We hypothesize that ROS work in concert with unfaithful DNA repair mechanisms to introduce mutations into the sequence encoding BCR/ABL kinase. Although ROS appear to play a major role in BCR/ABL-mediated mutagenesis, other factors may be important, too. For example, DNA polymerase $\beta$ is overexpressed in BCR/ABL cells, which may diminish the fidelity of BER and HRR [46]. Altogether, we postulate that excessive oxidative DNA damage and unfaithful DNA repair mechanisms contribute to point mutations leading to resistance to IM and other small-molecule inhibitors.

In conclusion, we postulate that reciprocal chromosomal translocations carrying the $B C R / A B L$ gene result from unfaithful repair of DNA DSBs. BCR/ABL kinase, the product of a hybrid gene, is able to initiate malignant transformation and further accelerate genomic instability, leading to malignant progression of the disease, and can induce resistance to genotoxic treatment and kinasetargeted small-molecule inhibitors.

\section{Conclusions}

The $\mathrm{t}(9 ; 22)$ reciprocal chromosomal translocation results from faulty repair of DNA DSBs, so it could be postulated that appearance of the translocation is a consequence of a pre-existing condition of genomic instability. However, the presence of BCR/ABL kinase is associated with elevation of DNA damage induced by ROS or genotoxic agents. Leukemia cells are protected from apoptosis by $\mathrm{BCR} / \mathrm{ABL}$ kinase and are capable of repairing more DNA lesions, but the repair is usually unfaithful. Therefore, BCR/ABL-positive cells, which survive genotoxic stress, may carry additional genetic errors leading to resistance to IM and malignant progression of the disease. CML cells thus display mutator phenotype.

\section{References and Recommended Reading}

Papers of particular interest, published recently, have been highlighted as:

- Of importance

$\bullet \quad$ Of major importance

1. Robinson DR, Wu YM, Lin SF: The protein tyrosine kinase family of the human genome. Oncogene 2000, 19:5548-5557.

2. Blume-Jensen P, Hunter T: Oncogenic kinase signalling. Nature 2001, 411:355-365.

3. Kolibaba KS, Druker BJ: Protein tyrosine kinases and cancer. Biochim Biophys Acta 1997, 1333:F217-F248.

4. Kurzrock R, Kantarjian HM, Druker BJ, Talpaz M: Philadelphia chromosome-positive leukemias: from basic mechanisms to molecular therapeutics. Ann Intern Med 2003, 138:819-830.
5. Bartkova J, Horejsi Z, Koed K, et al.: DNA damage response as a candidate anti-cancer barrier in early human tumorigenesis. Nature 2005, 434:864-870.

6. Gorgoulis VG, Vassiliou LV, Karakaidos P, et al.: Activation of the DNA damage checkpoint and genomic instability in human precancerous lesions. Nature 2005, 434:907-913. Cells display extensive DNA damage associated with activation of cell cycle checkpoints before the malignancy occurs. The transformation process is associated with disassembly of the checkpoint machinery and DNA errors.

7. Aplan PD: Causes of oncogenic chromosomal translocation. Trends Genet 2006, 22:46-55.

8. Richardson C, Jasin M: Frequent chromosomal translocations induced by DNA double-strand breaks. Nature 2000, 405:697-700.

9. Beckman KB, Ames BN: Oxidative decay of DNA. J Biol Chem 1997, 272:19633-19636.

10. Karanjawala ZE, Murphy, N, Hinton, DR, et al.: Oxygen metabolism causes chromosome breaks and is associated with the neuronal apoptosis observed in DNA double-strand break repair mutants. Curr Cell Biol 2002, 12:397-402.

11. Vafa O, Wade M, Kern S, et al.: c-Myc can induce DNA damage, increase reactive oxygen species, and mitigate p53 function: a mechanism for oncogene-induced genetic instability. Mol Cell 2002, 9:1031-1044.

12. Yu TW, Anderson D: Reactive oxygen species-induced DNA damage and its modification: a chemical investigation. Mutat Res 1997, 379:201-210.

13. Slupphaug G, Kavli B, Krokan HE: The interacting pathways for prevention and repair of oxidative DNA damage. Mutat Res 2003, 531:231-251.

14. Limoli CL, Giedzinski E, Morgan WF, et al.: Persistent oxidative stress in chromosomally unstable cells. Cancer Res 2003, 63:3107-3111.

15. Abeysinghe SS, Chuzhanova N, Krawczak M, et al.: Translocation and gross deletion breakpoints in human inherited disease and cancer I: nucleotide composition and recombination-associated motifs. Hum Mutat 2003, 22:229-244.

16. Akman SA, O'Connor TR, Rodriguez H: Mapping oxidative DNA damage and mechanisms of repair. Ann N Y Acad Sci 2000, 899:88-102.

17. Strick R, Zhang Y, Emmanuel N, Strissel PL: Common chromatin structures at breakpoint cluster regions may lead to chromosomal translocations found in chronic and acute leukemias. Hum Genet 2006, 119:479-495.

18. Preston DL, Kusumi S, Tomonaga M, et al.: Cancer incidence in atomic bomb survivors. Part III. Leukemia, lymphoma and multiple myeloma, 1950-1987. Radiat Res 1994, 137:S68-S97.

19. Martinelli G, Terragna C, Amabile M, et al.: Alu and translisin recognition site sequences flanking translocation sites in a novel type of chimeric bcr-abl transcript suggest a possible general mechanism for bcr-abl breakpoints. Haematologica 2000, 85:40-46.

20. Bassing CH, Alt FW: The cellular response to general and programmed DNA double strand breaks. DNA Repair (Amst) 2004, 3:781-796.

21.• Weinstock DM, Elliott B, Jasin M: A model of oncogenic rearrangements: differences between chromosomal translocation mechanisms and simple double-strand break repair. Blood 2006, 107:777-780.

Nonhomologous end-joining appears to be responsible for the majority of chromosomal translocations.

22. Papadopoulos PC, Greenstein AM, Gaffney RA, et al.: Characterization of the translocation breakpoint sequences in Philadelphia-positive acute lymphoblastic leukemia. Genes Chromosomes Cancer 1990, 1:233-239.

23.• Elliott B, Richardson C, Jasin M: Chromosomal translocation mechanisms at intronic Alu elements in mammalian cells. Mol Cell 2005, 17:885-894.

When DNA double-strand breaks were induced adjacent to identical Alu elements, translocations arose from single-strand annealing. With diverged $A l u$ elements, nonhomologous end-joining predominated as the translocation mechanism. 
24. Arlinghaus R, Sun T: Signal transduction pathways in Bcr-Abl transformed cells. Cancer Treat Res 2004, 119:239-270.

25. Skorski T: BCR/ABL regulates response to DNA damage: the role in resistance to genotoxic treatment and in genomic instability. Oncogene 2002, 21:8591-8604.

26. Nieborowska-Skorska M, Stoklosa T, Datta M, et al.: ATR-Chk1 axis protects BCR/ABL leukemia cells from the lethal effect of DNA double-strand breaks. Cell Cycle 2006, 5:994-1000.

27. Skorski T: Oncogenic tyrosine kinases and the DNA-damage response. Nat Rev Cancer 2002, 2:351-360.

28. Deininger M: Resistance to imatinib: mechanisms and management. J Natl Compr Canc Netw 2005, 3:757-768.

29.•• Nowicki MO, Falinski R, Koptyra M, et al.: BCR/ABL oncogenic kinase promotes unfaithful repair of the reactive oxygen species-dependent DNA double-strand breaks. Blood 2004, 104:3746-3753.

We showed that BCR/ABL kinase induces reactive oxygen species, which cause DNA double-strand breaks. These lesions are repaired by homologous recombination repair and nonhomologous endjoining. In leukemia cells, point mutations are introduced into the homologous recombination repair products and large deletions are introduced into the nonhomologous end-joining products.

30. Slupianek A, Nowicki MO, Koptyra M, Skorski T: BCR/ABL modifies the kinetics and fidelity of DNA doublestrand breaks repair in hematopoietic cells. DNA Repair (Amst) 2006, 5:243-250.

31. Keeshan K, Mills KI, Cotter TG, McKenna SL: Elevated Bcr-Abl expression levels are sufficient for a haematopoietic cell line to acquire a drug-resistant phenotype. Leukemia 2001, 15:1823-1833.

32. Canitrot $\mathrm{Y}$, Falinski R, Louat T, et al.: p210 BCR/ABL kinase regulates nucleotide excision repair (NER) and resistance to UV radiation. Blood 2003, 102:2632-2637.

33. Henning W, Sturzbecher HW: Homologous recombination and cell cycle checkpoints: Rad51 in tumour progression and therapy resistance. Toxicology 2003, 193:91-109.

34. Slupianek A, Hoser G, Majsterek I, et al.: Fusion tyrosine kinases induce drug resistance by stimulation of homologydependent recombination repair, prolongation of $G(2) / M$ phase, and protection from apoptosis. Mol Cell Biol 2002, 22:4189-4201.

35. Slupianek A, Schmutte C, Tombline G, et al.: BCR/ABL regulates mammalian RecA homologs, resulting in drug resistance. Mol Cell 2001, 8:795-806.

36. Deutsch E, Dugray A, Abdulkarim B, et al.: BCR-ABL down-regulates the DNA repair protein DNA-PKcs. Blood 2001, 97:2084-2090.

37. Brady N, Gaymes TJ, Cheung M, et al.: Increased errorprone NHEJ activity in myeloid leukemias is associated with DNA damage at sites that recruit key nonhomologous end-joining proteins. Cancer Res 2003, 63:1798-1805.

38. Gaymes TJ, Mufti GJ, Rassool FV: Myeloid leukemias have increased activity of the nonhomologous end-joining pathway and concomitant DNA misrepair that is dependent on the Ku70/86 heterodimer. Cancer Res 2002, 62:2791-2797.
39. Bedi A, Barber JP, Bedi GC, et al.: BCR-ABL-mediated inhibition of apoptosis with delay of G2/M transition after DNA damage: a mechanism of resistance to multiple anticancer agents. Blood 1995, 86:1148-1158.

40. Goldberg Z, Levav Y, Krichevsky S, et al.: Treatment of chronic myeloid leukemia cells with imatinib (STI571) impairs p53 accumulation in response to DNA damage. Cell Cycle 2004, 3:1188-1195.

41. Dierov J, Dierova R, Carroll M: BCR/ABL translocates to the nucleus and disrupts an ATR-dependent intra-S phase checkpoint. Cancer Cell 2004, 5:275-285.

42. Johansson B, Fioretos T, Mitelman F: Cytogenetic and molecular genetic evolution of chronic myeloid leukemia. Acta Haematol 2002, 107:76-94.

43. Calabretta B, Perrotti D: The biology of CML blast crisis. Blood 2004, 103:4010-4022.

Comprehensive review of CML progression from the chronic phase to blast crisis.

44. Thompson LH, Schild D: Homologous recombinational repair of DNA ensures mammalian chromosome stability. Mutat Res 2001, 477:131-153.

45.• Richardson C, Stark JM, Ommundsen M, Jasin M: Rad51 overexpression promotes alternative double-strand break repair pathways and genome instability. Oncogene 2004, 23:546-553.

Overexpression of Rad51, a key element in homologous recombination repair, causes chromosomal instability.

46. Canitrot Y, Lautier D, Laurent G, et al.: Mutator phenotype of BCR-ABL transfected $\mathrm{Ba} / \mathrm{F} 3$ cell lines and its association with enhanced expression of DNA polymerase beta. Oncogene 1999, 18:2676-2680.

47. McGill CB, Holbeck SL, Strathern JN: The chromosome bias of misincorporations during double-strand break repair is not altered in mismatch repair-defective strains of Saccharomyces cerevisiae. Genetics 1998, 148:1525-1533.

48. Lieber MR, Ma Y, Pannicke U, Schwarz K: Mechanism and regulation of human non-homologous DNA end-joining. Nat Rev Mol Cell Biol 2003, 4:712-720.

49. Druker BJ, Tamura S, Buchdunger E, et al.: Effects of a selective inhibitor of the Abl tyrosine kinase on the growth of Bcr-Abl positive cells. Nat Med 1996, 2:561-566.

$50 . \bullet$ Koptyra M, Falinski R, Nowicki MO, et al.: BCR/ABL kinase induces self-mutagenesis via reactive oxygen species to encode imatinib resistance. Blood 2006, 108:319-327.

$\mathrm{BCR} / \mathrm{ABL}$ kinase elevates reactive oxygen species, which cause oxidative DNA damage in BCR/ABL-positive leukemia cells. This mechanism contributes to mutagenesis in the kinase domain leading to the resistance to imatinib mesylate. Antioxidants reduce mutagenesis and appearance of leukemia clones resistant to imatinib. 